\title{
Unsupervised Domain Adaptation Techniques based on Auto-encoder for Non-stationary EEG-based Emotion Recognition
}

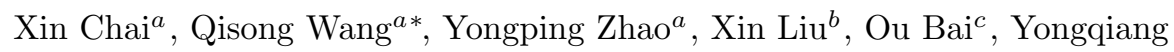 \\ $\mathrm{Li}^{a}$ \\ ${ }^{a}$ School of Electrical Engineering and Automation, Harbin Institute of Technology, Harbin, \\ China \\ ${ }^{b}$ Department of Traffic Information and Control Engineering, School of Transportation \\ Science and Engineering, Harbin Institute of Technology, Harbin, China \\ ${ }^{c}$ Department of Electrical and Computer Engineering, Florida International University, \\ $U S A$
}

\begin{abstract}
In electroencephalography (EEG)-based emotion recognition systems, the distribution between the training samples and the testing samples may be mismatched if they are sampled from different experimental sessions or subjects because of user fatigue, different electrode placements, varying impedances, etc. Therefore, it is difficult to directly classify the EEG patterns with a conventional classifier. The domain adaptation method, which is aimed at obtaining a common representation across training and test domains, is an effective method for reducing the distribution discrepancy. However, the existing domain adaptation strategies either employ a linear transformation or learn the nonlinearity mapping without a consistency constraint; they are not sufficiently powerful to obtain a similar distribution from highly non-stationary EEG signals. To address this problem, in this paper, a novel component, called the subspace alignment auto-encoder (SAAE), is proposed. Taking advantage of both nonlinear transformation and a consistency constraint, we combine an auto-encoder network and a subspace alignment solution in a unified framework. As a result,
\end{abstract}

\footnotetext{
* Corresponding author

Email address: wangqisong@hit.edu.cn;11b901011@hit.edu.cn (Qisong Wang ${ }^{a}$ )
}

Preprint submitted to Journal of Computers in Biology and Medicine

October 17, 2016

C) 2016. This manuscript version is made available under the Elsevier user license http://www.elsevier.com/open-access/userlicense/1.0/ 
the source domain can be aligned with the target domain together with its class label, and any supervised method can be applied to the new source domain to train a classifier for classification in the target domain, as the aligned source domain follows a distribution similar to that of the target domain. We compared our SAAE method with six typical approaches using a public EEG dataset containing three affective states: positive, neutral, and negative. Subject-to-subject and session-to-session evaluations were performed. The subject-to-subject experimental results demonstrate that our component achieves a mean accuracy of $77.88 \%$ in comparison with a state-of-the-art method, TCA, which achieves $73.82 \%$ on average. In addition, the average classification accuracy of SAAE in the session-to-session evaluation for all the 15 subjects in a dataset is $81.81 \%$, an improvement of up to $1.62 \%$ on average as compared to the best baseline TCA. The experimental results show the effectiveness of the proposed method relative to state-of-the-art methods. It can be concluded that SAAE is a useful and effective tool for decreasing domain discrepancy and reducing performance degradation across subjects and sessions in the EEG-based emotion recognition field.

Keywords: Emotion recognition, EEG, domain adaptation, Auto-encoder, MMD

\section{Introduction}

Affective computing, which can combine emotion with human-computer interaction, has been vigorously investigated in recent years. One of the most important elements in affective computing, emotion recognition, is aimed at 5 creating affective user interfaces between humans and computers and helping determine the psychiatric health of patients. A variety of measures have been used for emotion recognition, among which electroencephalography (EEG) signal methods [1] 2] have been attracting attention because of their objective evaluation and the signals' immediate responses to emotional stimuli, as com10 pared with facial expressions and gestures [3] [4]. To classify the EEG signals, 


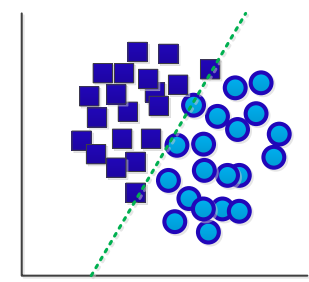

(a) Source Domain In Subject 1

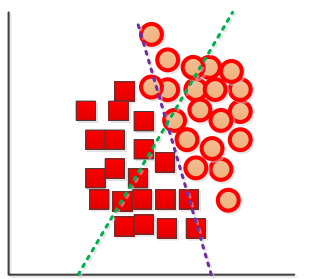

(b) Target Domain In Subject 2

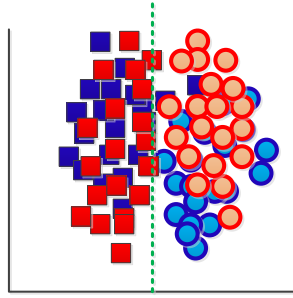

(c) Domain Adaptation

Figure 1: (a) Feature distribution for Subject 1. (b) Feature distribution for Subject 2. Classifiers trained on Subject 1 generalize poorly when applied to Subject 2, because of the distribution discrepancy. (c) Source and target domain after domain adaptation; the distribution discrepancy is reduced, and classifiers trained on Subject 1 generalize well when applied to Subject 2 .

useful features must be extracted using a suitable transformation of the data. Different classifiers have been implemented for multiclass EEG signal classification, such as the support vector machine (SVM), $k$-nearest neighbor (KNN), and linear regression (LR). Furthermore, in studies in the literature [6], deep belief networks (DBNs) have been used to build EEG-based emotion recognition models for three classes of emotion: positive, neutral, and negative. DBNs have achieved state-of-the-art performances. However, the above mentioned strategies involve training and testing the classifier for the same subject and even the same experimental session. For a conventional classification problem, the classifier must be learned on training samples with class labels and then applied to testing samples, based on the assumption that the training samples and testing samples share the same or similar distributions.

Thus far, it remains difficult to classify EEG patterns across subjects with a conventional classifier because of the variability between subjects and the high non-stationarity of EEG signals 7]. Fig 1 demonstrates a simple setting: in EEG-based emotion recognition systems, the distribution between the training and testing samples may be mismatched if they are sampled from different experimental sessions or subjects. Studies in the literature [8] 9] have proven that EEG signals are unstable in session-to-session and subject-to-subject transfers so because of user fatigue, differing electrode placement, varying impedances, etc. 
For one subject on a given day, well-trained classifiers can perform accurately, but usually the performance is degraded on subsequent days when using the same experimental setup. Thus far, most studies have focused on selecting features or channels [10, 11, 12, [13] and have neglected to consider distribution discrepancy between subjects.

In recent years, several machine learning techniques, referred to as domain adaptation techniques [14, have been investigated. Typically, training samples with class labels are defined as source domains and testing samples with different distributions are called target domains [15]. Domain adaptation is typically aimed at utilizing information from both the source and target domains during the learning process, and building classifiers robust to mismatched distributions, based on the assumption that both the source and target domains share the same task, as shown in Fig 1

In general, domain adaptation for EEG patterns faces two main challenges:

45 (1) reduction in the domain discrepancy, and (2) learning of common representations across training and test domains [16] [17] [18]. In [8, Sugiyama et al. applied a covariate shift adaptation method to a brain-computer interface (BCI) competition dataset to analyze non-stationary EEG signals in different sessions. They assumed that, although the marginal distribution between the subjects 50 or sessions is mismatched, the decision rule remains constant. To correct the covariate shifts, they utilized the Kullback-Leibler importance estimation procedure to re-weight the training data and correct the difference between different subjects or sessions. More widely used methods attempt to employ transformation to project the source and target domain onto a subspace. In previous work [19, a linear reconstruction strategy based on sparse coding was used in an attempt to modify the representation of both source and target data to obtain a well-aligned feature space; this technique achieves a good performance for computer vision problems such as object recognition. However, for EEG signals, because the source domain is quite different from the target domain,

60 linear transformation is not sufficiently powerful to obtain a similar distribution for the two domains 20] 21] 22]. Recently, auto-encoder networks, which 


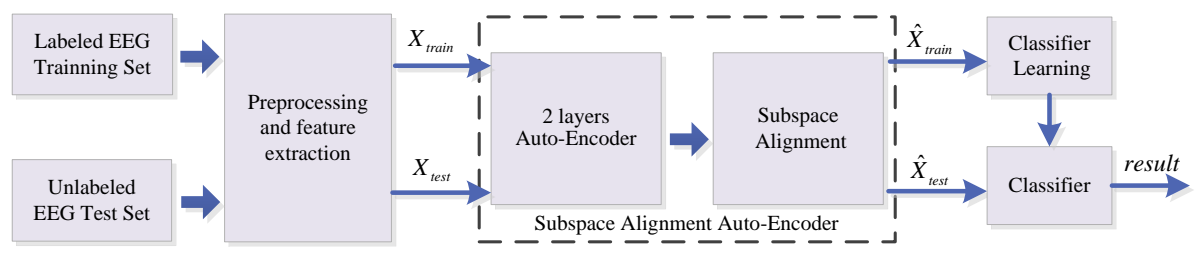

Figure 2: Flowchart of the electroencephalography emotion recognition system integrating the proposed subspace alignment auto-encoder method.

can learn a common representation across training and test domains, have been successfully applied for reducing the cross-domain discrepancy by utilizing the deep structures in the field, for example, face recognition and speech recognition 23 24] 25]. The nonlinearity embedded in the auto-encoder network makes it more effective and efficient when learning domain-invariant features between source and target data. However, these methods also face one challenge: because there is no consistency constraint, the auto-encoder network may, at times, unexpectedly increase the cross-domain distribution discrepancy for new deep features.

To solve the problems mentioned above, in this paper, a novel strategy called the subspace alignment auto-encoder (SAAE) is proposed. In the method, we take advantage of both the nonlinear transformation and the consistency constraint, and combine the auto-encoder network and the subspace alignment solution in a unified framework, as shown in Fig 2. In this study, by modeling each channel bandpower as a separate feature, an efficient feature called differential entropy (DE) was used to measure the complexity of EEG signals as the input of the SAAE model. In SAAE, first an unsupervised auto-encoder learns using both the source and target domain features; then, these features are transformed into a domain-invariant subspace using the well-trained auto-encoder to ensure the feasibility of shifting between domains. The second step is to apply the subspace alignment solution in the domain-invariant subspace. Specifically, we constrain the distribution discrepancy by minimizing the maximum mean discrepancy (MMD) 26] in an infinite-dimensional reproducing kernel Hilbert 
space (RKHS). As a result, the source domain can be aligned to a target domain together with its class label. Additionally, any supervised method can be applied in the new source domain to train a classifier for classification in the target domain, as the aligned source domain follows a distribution similar to that of the target domain. Note that our SAAE is an unsupervised strategy

90 without any labeled test data.

The major contributions of this research are as follows.

1) In contrast to the conventional auto-encoder network, we combine this method with a subspace alignment solution in the domain-invariant subspace to constrain the distribution discrepancy between the training and test domains.

2) Our study provides an evaluation and analysis of several state-of-the-art domain adaptation techniques in the field of pattern recognition for application to non-stationary EEG signal classification.

The remainder of this paper is organized as follows. In Section 2, we briefly introduce our experimental design, and then, preprocessing and feature extrac-

\section{Preliminaries}

\subsection{Experimental design}

To investigate the effectiveness of the domain adaptation algorithms proposed in this study, we evaluated our SAAE method using a publicly available emotion EEG dataset called the SJTU Emotion EEG Dataset (SEED) dataset ${ }_{110}^{1}[6]$. Unlike the subjects in the existing emotion EEG datasets, each subject who participated in creating SEED took part in three experimental sessions at 


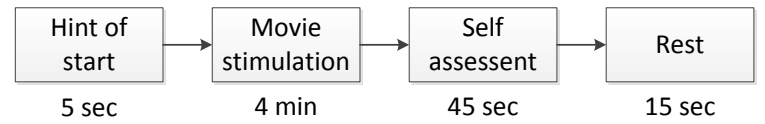

Figure 3: Protocol for the electroencephalography-based emotion recognition experiment(adapted from [6]).

intervals of one week or longer. Therefore, the SEED dataset is the best choice for investigating the influence of distribution discrepancy across both subjects and sessions for non-stationary EEG signal classification.

The SEED dataset consists of data for 15 subjects ( 8 females and 7 males; average age 23.27 years). Each subject was stimulated by Chinese film clips (because native-culture factors may affect elicitation in emotional experiments). For feedback, the participants were asked to assess their emotions in terms of positive, neutral, and negative feelings immediately after watching each of the selected film clips. As shown in Fig 3 , in total, 15 trials were conducted in each experiment, where the duration of each trial was approximately $4 \mathrm{~m}$. For each emotion (positive, neutral, and negative), there were five corresponding emotional clips. There is a $5 \mathrm{~s}$ hint before each clip and $45 \mathrm{~s}$ of feedback after each clip. In the SEED dataset, the EEG signals were recorded using a 64channel $\mathrm{AgCl}$ electrode cap (channels) at a sampling rate of 1,000 $\mathrm{Hz}$ using the international 10-20 system; the impendence of each electrode was less than 5k $\Omega$. More details about the SEED dataset are given in [6].

\subsection{Preprocessing and feature extraction}

The SEED dataset provides EEG signals that were down-sampled to $200 \mathrm{~Hz}$; all the EEG data were manually inspected to remove samples that had been seriously contaminated by electromyography (EMG) or electro-oculography (EOG). In addition, EOG signals were also recorded to identify blink artifacts. A bandpass filter between $0 \mathrm{~Hz}$ and $75 \mathrm{~Hz}$ was applied to preprocess the EEG data to filter noise and remove artifacts. There were, in total, approximately 3300 clean 135 epochs for one experiment in each channel, where the duration of each epoch was $1 \mathrm{~s}$ without overlap. 
According to the analysis results presented in [27] 28], a simple and efficient feature called differential entropy (DE) shows a balanced performance for EEGbased emotion recognition as compared with other features, such as differential asymmetry (DASM), rational asymmetry (RASM), and asymmetry (ASM). Because feature extraction was not the research emphasis of this study, we used the DE feature as an input for our domain adaptation model. DE is defined as

$$
h(X)=-\int_{-\infty}^{\infty} \frac{1}{\sqrt{2 \pi \sigma^{2}}} \exp \frac{(x-\mu)^{2}}{2 \sigma^{2}} \log \frac{1}{\sqrt{2 \pi \sigma^{2}}} \exp \frac{(x-\mu)^{2}}{2 \sigma^{2}} d x=\frac{1}{2} \log 2 \pi e \sigma^{2}
$$

where the time series $X$ obeys the Gauss distribution $N\left(\mu, \sigma^{2}\right)$. In this study, DE was calculated using the standard five frequency bands (delta: $1-3 \mathrm{~Hz}$, theta: 4-7 Hz, alpha: 8-13 Hz, beta: 14-30 Hz, gamma: 31-50 Hz). There are 310 dimensions of DE features in total, because each frequency-band signal has 62 channels.

\section{Proposed method}

\subsection{Problem definition and overall approach}

In this study, DE feature vectors extracted from samples in both the training and test sets were used as the input to our SAAE model. Specifically, feature vectors from the training set with class labels are defined as the source domain. In the source domain, there are $n s$ labeled samples in $d$ dimensions $(d=310)$, denoted as $X_{S}=\left[x_{s 1}, x_{s 2}, \ldots, x_{s n s}\right]$ with class labels $Y_{s}=\left[y_{s 1}, y_{s 2}, \ldots, y_{s n s}\right]$, where $x_{s i}$ is the DE feature of the $i-t h$ sample from the training set and $y_{s i}$ is its corresponding class label. Similarly, feature vectors extracted from the test set, which are denoted as $X_{T}=\left[x_{t 1}, x_{t 2}, \ldots, x_{t n t}\right]$, where $x_{t i}$ is the DE feature of the $i-t h$ sample from the test set, are defined as the target domain. Given a labeled source domain $X_{S}$ and an unlabeled target domain $X_{T}$, the problem is how to learn a new feature representation such that the distribution discrepancy 155 is reduced and to train an adaptive classifier for the target domain.

As shown in Fig 4, SAAE employs a three-step strategy. First, DE features 


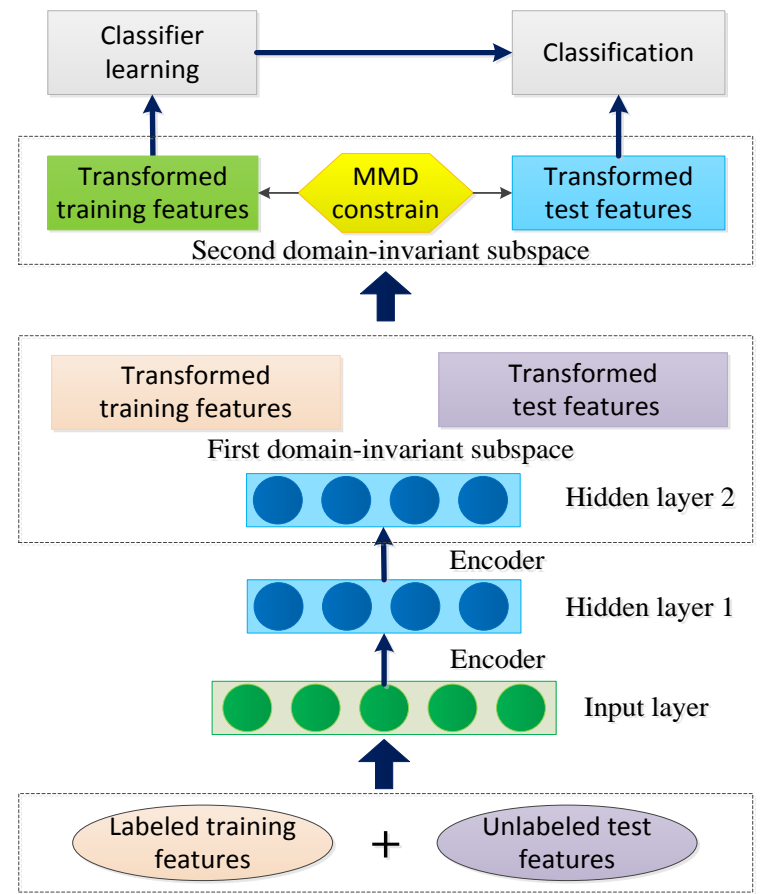

Figure 4: Overview of subspace alignment auto-encoder.

are transformed into a domain-invariant subspace using the well-trained stacked auto-encoder. In the second step, we constrain the distribution discrepancy by minimizing the MMD [26] on source instances in an infinite-dimensional RKHS. As a result, the source domain can be aligned with the target domain, including its class label, and any supervised method can be applied to the new source domain to train a classifier for classification in the target domain, because the aligned source domain follows a distribution similar to that of the target domain.

\subsection{Domain-invariant feature learning by stacked auto-encoder}

In this section, we introduce the conventional auto-encoder framework. The auto-encoder is a fully connected unsupervised learning neural network for learning a better representation of a dataset in pre-training for classification tasks by setting the target values to be equal to the inputs. The single hidden layer 


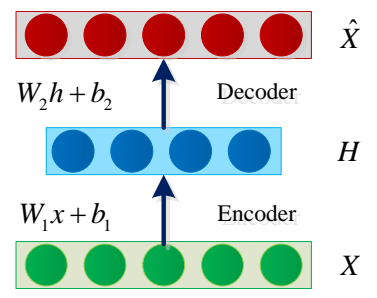

Figure 5: Architecture of basis auto-encoder.

auto-encoder given in Fig 5 typically has one input layer $x$, one output layer $\hat{x}$, and one hidden layer $h$; the connection between the input layer and hidden layer is called an encoder, and the connection between the hidden layer and the output layer is called a decoder. The encoder attempts to map the input $x$ onto the hidden representation $h(x)$ as

$$
h(\tilde{x})=f\left(w_{1} \cdot x+b_{1}\right)
$$

where $f$ is the logistic sigmoid function, $w_{1}$ is a weight matrix, and $b_{1}$ is a bias vector for hidden nodes. The decoder attempts to map the hidden representation $h(x)$ back onto the reconstruction $\hat{x}$ as

$$
\hat{x}=f\left(w_{2} \cdot h(x)+b_{2}\right)
$$

where $w_{2}$ is a weight matrix and $b_{2}$ is a bias vector for the output layer. Given the training samples, to optimize the parameters $w_{1}, b_{1}, w_{2}$, and $b_{2}$, the autoencoder is typically trained to minimize some form of reconstruction error loss. Typically, the objective function is given by

$$
\min _{w_{1}, b_{1}, w_{2}, b_{2}} \sum_{i=1}^{N}\left\|x_{i}-\hat{x}_{i}\right\|
$$

In this study, limited-memory BFGS (L-BFGS) 29] was employed for parameter estimation. When an auto-encoder has been trained, its parameters describe a new representation for $X_{S}$, and $X_{T}$ can be used to directly feed a classifier. 
The conventional auto-encoder in Fig 5 attempts to reconstruct the input

Eventually, the new higher-level representation of $X_{H S}$ can be used to directly train a conventional classifier on the source domain. This classifier can then be used to test the new higher-level representation for $X_{H T}$ on the test domain.

\subsection{Subspace alignment}

Although the auto-encoder-based deep learning method can efficiently learn the domain-invariant representation between the training and test data to overcome the adverse effects of the non-stationarity of EEG signals, this approach still suffers from one limitation. Because there is no consistency constraint, the auto-encoder network may unexpectedly enlarge the cross-domain distribution discrepancy for the new deep features and occasionally lead to statistically unbounded target errors. Therefore, to minimize the distribution difference between the two domains, the second step of our method attempts to achieve subspace alignment by employing an effective constraint. After transforming the DE features of EEG signals into a domain-invariant subspace using the auto-encoder, the new representation is used to learn another space, in which the distance between the empirical expectations of the source and target data is minimized. Recently, an effective nonparametric distance estimation method named MMD was designed in which the distributions are embedded in an 
RKHS. In this study, we adopted this technique as the distance constraint in the RKHS to minimize the distribution difference between the two domains. Define $X_{H S}=\left[x_{h s 1}, x_{h s 2}, \ldots, x_{h s n s}\right] \in R^{m \times n s}$ as the feature transformed from the training data by the stacked auto-encoder, and $X_{H T}=\left[x_{h t 1}, x_{h t 2}, \ldots, x_{h t n t}\right] \in R^{m \times n t}$ as the feature transformed from the test data. If we map the feature into a new space using a universal kernel $\varphi$, the distance between the empirical means of the two domains can be used to measure the distance between the two distributions $X_{H S}$ and $X_{H S}$ :

$$
M M D\left(X_{H S}, X_{H T}\right)=\left\|\frac{1}{n s} \sum_{i=1}^{n s} \varphi\left(x_{h n i}\right)-\frac{1}{n t} \sum_{i=1}^{n t} \varphi\left(x_{h t i}\right)\right\|_{H}^{2}
$$

where $\|\cdot\|_{H}$ is the RKHS norm. In this study, we utilized kernel-PCA as the feature transformation $\varphi$. On the one hand, the features that are not helpful for domain adaptation classification can be removed; on the other hand, we can work in a possibly infinite-dimensional feature space. We set the kernel mapping $\varphi(X)=\left[\varphi\left(X_{1}\right), \ldots, \varphi\left(X_{n}\right)\right]$ and the kernel matrix $K=\varphi(X)^{T} \varphi(X)$. The goal of kernel-PCA is to find a transformation matrix $A$ such that the embedded data variance is maximized. Therefore, we utilize the representer theorem for kernel-PCA:

$$
\max _{A^{T} A=I} \operatorname{tr}\left(A^{T} K H K^{T} A\right)
$$

where $H=\mathbf{I}-\frac{1}{n} \mathbf{1}$ is the centering matrix, $n=n s+n t, \mathbf{I}$ is the identity matrix and $\mathbf{1}$ is the $n \times n$ matrix of ones. $A$ is the transformation matrix for kernel-PCA and the new subspace features become $Z=A^{T} K$. In addition, MMD computes the distance between the empirical expectations of the source and target data as

$$
\left\|\frac{1}{n s} \sum_{i=1}^{n s} A^{T} K_{i}-\frac{1}{n t} \sum_{j=n s+1}^{n s+n t} A_{H}^{T} K_{j}\right\|_{H}^{2}=\operatorname{tr}\left(A^{T} K M K^{T} A\right)
$$


where $M$ is the MMD matrix, which is computed as

$$
M_{i j}=\left\{\begin{array}{c}
\frac{1}{n_{s} n_{s}}, x_{i}, x_{j} \in X_{S} \\
\frac{1}{n_{t} n_{t}}, x_{i}, x_{j} \in X_{T} \\
\frac{-1}{n_{s} n_{t}}, \text { otherwise }
\end{array}\right.
$$

To respect the intrinsic geometric structure underlying the input data, a graph regularization is also added into the objective function. Define $W$ as the weight matrix of $X$, and $d_{i}=\sum_{j=1}^{n} W_{i j}, D=\operatorname{diag}\left(d_{1}, \ldots, d_{n}\right)$. The graph Laplacian can be expressed as $L=D-W[22$. By minimizing Eq7 the objective function can be expressed as

$$
\min _{A^{T} K H K^{T} A=I} \operatorname{tr}\left(A^{T} K S K^{T} A\right)+\lambda t r\left(A^{T} A\right)
$$

where $S=M+L$, and the regularization term $\operatorname{tr}\left(A^{T} A\right)$ is used to control the complexity of $A$. If we define $\Phi$ as the Lagrange multiplier, the Lagrange function of $\mathrm{Eq} 9$ can be expressed as

$$
L=\operatorname{tr}\left(A^{T}\left(K S K^{T}+\lambda I\right) A\right)+\operatorname{tr}\left(\left(I-A^{T} K H K^{T} A\right) \Phi\right)
$$

Setting the derivative of $L$ to zero, we obtain the generalized eigen-decomposition:

$$
\left(K S K^{T}+\lambda I\right) A=K H K^{T} A \Phi
$$

185

\footnotetext{
$\left(K S K^{T}+\lambda I\right)^{-1} K H K^{T}$. Note that we developed our subspace alignment part
} based on the MMD constraint so that it is similar to that in [30].

\section{Results}

\subsection{Evaluation strategy}

190

In this study, we evaluated the proposed method on the SEED dataset. Note that SEED consists of the data of 15 subjects, who each participated in three 
experimental sessions; the interval between two sessions was one week or longer. Therefore, we evaluated our SAAE model in two scenarios: 1) subject-to-subject evaluation, in which the training and test domains were from different subjects; and 2) session-to-session evaluation, in which the source and target domains were from the same subject but different experimental sessions. To evaluate the efficacy, we systematically compared the proposed SAAE model with several state-of-the-art domain adaptation methods for EEG-based emotion recognition. First, we trained a linear SVM on the labeled source domain without applying domain adaptation technology, as a baseline. In addition, LR was also tested as a baseline, because we chose it as the base classifier. We systematically compared six state-of-the-art domain adaptation and deep learning methods for EEG-based emotion recognition.

- Support vector machine (SVM);

- Logistic regression (LR);

- Geodesic flow kernel (GFK) [31;

- Transfer sparse coding (TSC) [22];

- Transfer component analysis (TCA) [30];

- Auto-encoder (AE) 23;

- Subspace alignment (SA) without auto-encoder.

The linear SVM and LR were trained on the DE features of the source domain using LIBSVM [32] and LIBLINEAR software [33] as baselines. To provide an analysis of the state-of-the-art domain adaptation techniques when applied to non-stationary EEG signal classification, we also investigated GFK [31, TSC 22], TCA [30, and AE 23], which produce state-of-the-art performances in various domains for solving computer vision problems. For all methods, LR was chosen as the base classifier for fair comparison. Note that we further compared our SAAE with the graph regularized extreme learning machine (GELM) [34] on the session-to-session task groups. GELM has been shown to produce a 220 state-of-the-art performance on the SEED dataset; therefore, we used the results reported in the original paper 34 to provide a fair comparison.

Table 1 shows the parameters of the different methods. In this study, the 
SVM used a linear kernel, and the parameter $C$ was set by searching $\{0.01$, $0.05,0.1,0.5,1,5,10,50,100\}$ to find the optimal value. For LR, we tuned the parameter $C$ in $\{0.01,0.05,0.1,0.5,1,5,10,50,100\}$. For GFK, the dimension of the source subspace and target subspace was set at 64 . TSC is a domain adaptation method based on sparse coding. In this study, the number of basis vectors in the dictionary was set to 128 , sparsity regularization to 0.1 , and the number of iterations per TSC to 15. For the subspace-learning method TCA, we set the subspace bases as 60 , and the adaptation regularization parameter $\lambda$ was set by searching $\lambda \in\{0.01,0.1,1,10,100\}$. For the conventional auto-encoder, we set the structure to have two hidden layers; the number of hidden neurons was set by searching $\{50,100,200,400,600,1000\}$. For our SAAE, we also constructed an auto-encoder with two hidden layers, and an LR classifier was used for supervised learning for classification. For all experiments, the optimal number of hidden neurons was set by searching $\{50,100,200,400,600,1000\}$. For the subspace alignment part, the regularization parameter $\lambda$ was set by searching $\lambda \in\{0.01,0.1,1,10,100\}$; our results showed that 1.0 gives the highest average accuracy on all tasks.

\subsection{Subject-to-subject experimental results}

The non-stationarity of EEG signals across different subjects causes a mismatch in the feature distributions between the training and testing samples. Therefore, our emotion recognition model is aimed at learning new features to reduce the cross-subject discrepancy. To evaluate our SAAE model, first, we adopted a subject-to-subject strategy, where the training and test domains were taken from different subjects. We constructed 14 task groups for classification from each session. In each repetition, we fixed the data of Subject 1 as the training data and separated the data of the remaining 14 subjects as the test data, as shown in Table 2 .

Table 2 shows the evaluation results of the different methods for a total of 14 task groups. As seen from these comparisons, the standard classifiers, i.e., SVM and LR, performed poorly with a mean accuracy of $39.93 \%$ and $44.08 \%$, because 


\begin{tabular}{cc}
\hline Method & Parameter details \\
\hline SVM & $\begin{array}{c}\text { Linear kernel, the parameter C was set by } \\
\text { searching }\{0.01,0.05,0.1,0.5,1,5,10,50,100\} ;\end{array}$ \\
\hline LR & $\begin{array}{c}\text { We set the parameter C by searching } \\
\{0.01,0.05,0.1,0.5,1,5,10,50,100\} ;\end{array}$ \\
\hline GFK & Subspace dimension was set as $64 ;$ \\
\hline \multirow{2}{*}{ TSC } & Number of basis vectors was $128 ;$ \\
& Sparsity regularization was $0.1 ;$ \\
& Number of iterations per TSC was set as $15 ;$ \\
TCA & Subspace bases was set as $60 ;$ \\
& Regularizer was set by \\
& searching $\lambda \in\{0.01,0.1,1,10,100\} ;$ \\
AE & Structure with 2 hidden layers; \\
& The number of hidden neurons was set by \\
& searching $\{50,100,200,400,600,1000\} ;$ \\
\hline \multirow{2}{*}{ SA } & Subspace bases was set as $60 ;$ \\
& Regularizer was set by \\
& searching $\lambda \in\{0.01,0.1,1,10,100\} ;$ \\
& Structure with 2 hidden layers; \\
SAAE & The number of hidden neurons was set by \\
& searching $\{50,100,200,400,600,1000\} ;$ \\
& Subspace dimension was set as $60 ;$ \\
& Subspace regularization was set as $1 ;$
\end{tabular}

Table 1: Details of parameters used in different methods

no domain adaptation strategy was employed. Unexpectedly, GFK, which is a PCA-based domain adaptation method, also achieved a poor performance. We assume that the low-dimensional PCA subspaces may not accurately represent input data when the EEG features are complex. As expected, TCA and TSC, which are state-of-the-art domain adaptation methods, achieved a much better performance at $55.25 \%$ and $55.85 \%$ accuracy, respectively, as both methods attempt to minimize the distribution divergence between the training and test EEG features with a given constraint. The AE method performed slightly better than SVM, LR, and GFK, but worse than TCA and TSC; this may be because the AE network increased the distribution discrepancy for the new deep features with no consistency constraint. The SA without auto-encoder performed slightly better than TCA. As compared with these methods, our SAAE method achieved comparable accuracies with a mean accuracy of $62.50 \%$, as the deep structure of the auto-encoder coupled with subspace constraint with MMD can accurately minimize the distribution divergence between the training and test EEG features. It is worth mentioning that our SAAE method outperformed 


\begin{tabular}{ccccccccl}
\hline $\begin{array}{c}\text { Task } \\
\text { No. }\end{array}$ & SVM & LR & GFK & TSC & TCA & AE & SA & SAAE \\
\hline $1 \rightarrow 2$ & 44.16 & 36.20 & 44.08 & 53.41 & 44.53 & 45.25 & 40.32 & $\mathbf{5 5 . 6 4}$ \\
$1 \rightarrow 3$ & 41.45 & 55.36 & 45.07 & 58.98 & 68.97 & 45.25 & $\mathbf{7 2 . 2 7}$ & 67.39 \\
$1 \rightarrow 4$ & 33.00 & 43.24 & 34.79 & 64.43 & 69.92 & 50.94 & $\mathbf{7 4 . 1 6}$ & 73.04 \\
$1 \rightarrow 5$ & 38.57 & 29.66 & 32.88 & 41.74 & 40.97 & 37.53 & 38.67 & $\mathbf{4 3 . 8 4}$ \\
$1 \rightarrow 6$ & 37.93 & 57.54 & 39.48 & 57.80 & 62.88 & 50.04 & 56.52 & $\mathbf{6 4 . 6 9}$ \\
$1 \rightarrow 7$ & 40.56 & 43.04 & 38.59 & 52.30 & 55.68 & 47.89 & 58.82 & $\mathbf{6 0 . 3 6}$ \\
$1 \rightarrow 8$ & 43.86 & 48.90 & 47.87 & 47.89 & 50.60 & 43.18 & 58.93 & $\mathbf{6 6 . 7 7}$ \\
$1 \rightarrow 9$ & 36.78 & 46.62 & 36.71 & 51.89 & 48.67 & 42.69 & 54.49 & $\mathbf{6 4 . 4 9}$ \\
$1 \rightarrow 10$ & 30.16 & 44.68 & 36.44 & 56.60 & 50.45 & 43.95 & $\mathbf{5 7 . 5 0}$ & 49.03 \\
$1 \rightarrow 11$ & 48.29 & 44.23 & 40.52 & 63.53 & 62.37 & 44.62 & 62.57 & $\mathbf{6 5 . 8 8}$ \\
$1 \rightarrow 12$ & 37.96 & 33.52 & 58.52 & 53.82 & 52.74 & 44.40 & 48.61 & $\mathbf{6 4 . 8 3}$ \\
$1 \rightarrow 13$ & 38.65 & 44.91 & 44.17 & 55.11 & 53.38 & 48.11 & 48.08 & $\mathbf{5 8 . 1 0}$ \\
$1 \rightarrow 14$ & 49.50 & 46.07 & 40.18 & 55.95 & 56.86 & 44.41 & 59.78 & $\mathbf{6 9 . 7 2}$ \\
$1 \rightarrow 15$ & 38.15 & 43.13 & 41.09 & 68.45 & 55.46 & 41.33 & 59.25 & $\mathbf{7 1 . 1 6}$ \\
Average & 39.93 & 44.08 & 41.46 & 55.85 & 55.25 & 45.79 & 56.43 & $\mathbf{6 2 . 5 0}$ \\
\hline
\end{tabular}

Table 2: Classification accuracy (\%) of subject-to-subject task groups by fixing subject 1 as the training data

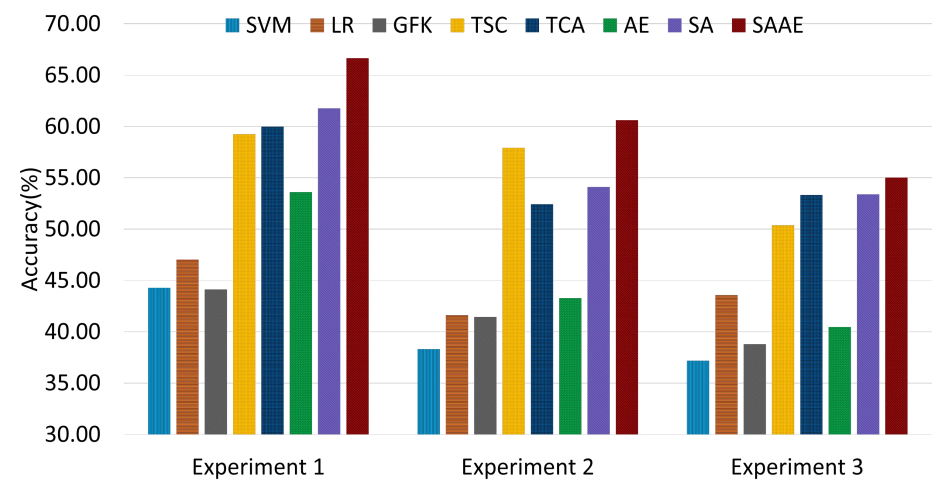

Figure 6: Average classification accuracy of the 14 task groups for every experimental session by fixing subject 1 as the training data.

the comparison methods for most task groups, which confirms that SAAE can perform stably.

In addition, to improve the interpretation, we also provide the evaluation average results of the 14 task groups for each experimental session in Fig 6 the interval between two sessions was at least one week. As these comparisons show, our SAAE method also achieved the best accuracies of all the methods.

To further confirm the effectiveness and stability of our method, the leaveone-subject-out cross validation method was adopted for the evaluation. In each repetition, the data from one subject were the test data and the data from 


\begin{tabular}{cccccccccc}
\hline $\begin{array}{c}\text { Subject } \\
\text { No. }\end{array}$ & SVM-ALL & SVM & LR & GFK & TSC & TCA & AE & SA & SAAE \\
\hline 1 & 77.06 & 44.16 & 49.86 & 47.89 & 64.74 & 69.31 & 57.04 & 74.12 & $\mathbf{7 8 . 6 3}$ \\
2 & 71.60 & 41.68 & 43.50 & 50.62 & $\mathbf{7 8 . 3 1}$ & 69.47 & 45.53 & 68.32 & 72.36 \\
3 & 72.52 & 46.10 & 51.14 & 47.85 & 63.95 & 72.43 & 62.20 & 74.20 & $\mathbf{7 9 . 5 0}$ \\
4 & 72.56 & 65.66 & 69.93 & 50.35 & 83.24 & 80.66 & 84.70 & 79.38 & $\mathbf{9 2 . 9 8}$ \\
5 & $\mathbf{6 6 . 6 7}$ & 44.34 & 49.34 & 36.92 & 51.44 & 57.78 & 51.63 & 60.51 & 65.41 \\
6 & $\mathbf{7 7 . 6 3}$ & 50.89 & 53.26 & 34.62 & 64.72 & 73.69 & 56.54 & 75.10 & 75.17 \\
7 & 69.32 & 55.36 & 58.54 & 35.97 & 67.64 & 75.02 & 64.01 & 79.33 & $\mathbf{7 9 . 3 6}$ \\
8 & 72.78 & 46.03 & 53.02 & 50.94 & 65.45 & 69.05 & 61.66 & 72.54 & $\mathbf{7 4 . 8 7}$ \\
9 & 74.33 & 55.20 & 54.53 & 51.43 & 74.64 & 82.62 & 56.02 & 83.13 & $\mathbf{8 8 . 8 4}$ \\
10 & 56.02 & 38.11 & 39.56 & 43.01 & 67.38 & 67.63 & 50.15 & 66.70 & $\mathbf{6 9 . 5 4}$ \\
11 & 70.97 & 49.30 & 64.68 & 46.36 & 73.82 & $\mathbf{8 4 . 0 7}$ & 65.59 & 82.15 & 81.49 \\
12 & 66.06 & 64.40 & 63.21 & 46.10 & 72.44 & 72.00 & 66.75 & 73.92 & $\mathbf{7 4 . 6 1}$ \\
13 & 73.13 & 74.16 & 69.89 & 51.75 & 75.28 & 80.39 & 69.82 & 80.81 & $\mathbf{8 1 . 3 1}$ \\
14 & 77.84 & 62.75 & 66.40 & 40.86 & 77.81 & 84.39 & 61.57 & $\mathbf{8 6 . 5 9}$ & 83.73 \\
15 & 61.52 & 55.43 & 47.75 & 43.13 & 64.55 & 68.85 & 53.76 & 64.27 & $\mathbf{7 0 . 3 1}$ \\
Average & 70.67 & 53.06 & 55.64 & 45.19 & 69.69 & 73.82 & 60.46 & 74.74 & $\mathbf{7 7 . 8 8}$ \\
\hline
\end{tabular}

Table 3: Classification accuracy (\%) of each subject using leave-one-subject-out cross validation method

the remaining 14 subjects were the training data. It is worth mentioning that it is infeasible to include all the data (about 47516 samples) from 14 subjects as the training data because of the limits of the memory for TCA, TSC, and our SAAE. Therefore, we randomly selected a subset of samples (300 samples) from each subject, and obtained 4200 samples in total as training data. All the data from one subject (about 3394 samples) were included as the test data. To comprehensively compare the effect of our method, we also concatenated all the data (about 47516 samples) from 14 subjects as the training data to train a classifier with SVM. The evaluation results are shown in the first column of Table 3

The classification (emotion recognition) accuracies of our SAAE method and the eight baseline methods are illustrated in Table 3 To provide a more accurate interpretation, we also show the average evaluation results of the 15 subjects for each experimental session in Fig 7. It is important to note that in the SVM-ALL method in the first column the classifier was trained by all 47516 samples, and in the other methods was trained by 4200 samples, which were randomly selected. We observe that the performance of SVM-ALL and SVM are improved greatly as compared with the person-to-person task, be- 


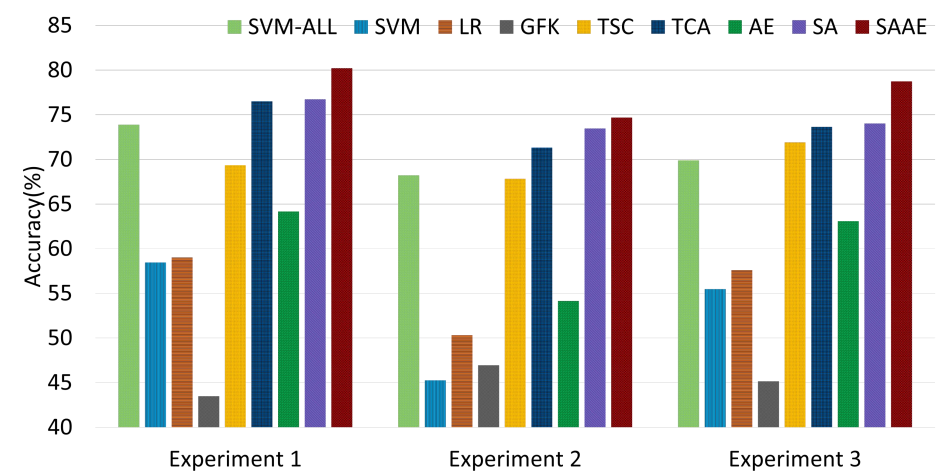

Figure 7: Average classification accuracy of the 15 task groups for each experimental session using leave-one-subject-out cross validation method.

cause the number of the training data was increased significantly. As expected, although there were only 4200 training data samples in total, TCA and TSC still achieved a better accuracy performance, $73.82 \%$ and $69.69 \%$, respectively. The AE method performed worse than TCA and TSC, and the SA without an auto-encoder slightly outperformed TCA. The average classification accuracy of SAAE for all 15 subjects was $77.88 \%$, an improvement of up to $4.06 \%$ on average as compared to the accuracy of the best baseline TCA. Although our SAAE was trained by only 4200 samples, it still outperformed the other approaches with the highest accuracy, and achieved a significant improvement as compared with the SVM-ALL, which was trained by all the 47516 samples. Fig 7 shows the average evaluation results of the 15 task groups for each experimental session. The results show that SAAE achieved the best accuracy of the methods, which confirms that SAAE can perform stably.

\subsection{Session-to-session experimental results}

As mentioned above, each subject in SEED participated in three experimental sessions, and the interval between sessions was at least one week. This experimental method can help us investigate whether the performance of our domain adaptation method can overcome the non-stationarity of EEG signals over time. The non-stationarity of EEG signals causes a mismatch in 


\begin{tabular}{cccccccccc}
\hline $\begin{array}{c}\text { Task group } \\
\text { No. }\end{array}$ & SVM & LR & GELM & GFK & TSC & TCA & AE & SA & SAAE \\
\hline $1 \rightarrow 2$ & 64.63 & 59.19 & 72.55 & 65.05 & 76.34 & 81.61 & 75.39 & 81.79 & $\mathbf{8 4 . 4 7}$ \\
$1 \rightarrow 3$ & 61.89 & 59.65 & 67.22 & 65.29 & 77.04 & 78.90 & 74.04 & 79.68 & $\mathbf{8 0 . 0 4}$ \\
$2 \rightarrow 1$ & 65.80 & 57.77 & 75.86 & 66.29 & 80.39 & 82.32 & 76.20 & 81.82 & $\mathbf{8 4 . 3 1}$ \\
$2 \rightarrow 3$ & 67.30 & 65.03 & 76.62 & 66.00 & 78.34 & 81.62 & 75.93 & 81.45 & $\mathbf{8 3 . 0 9}$ \\
$3 \rightarrow 1$ & 69.20 & 63.87 & 76.28 & 69.85 & 77.05 & 79.03 & 75.75 & 79.92 & $\mathbf{8 1 . 1 3}$ \\
$3 \rightarrow 2$ & 67.99 & 64.56 & 78.17 & 60.69 & 75.74 & 77.65 & 76.94 & 77.51 & $\mathbf{7 8 . 7 7}$ \\
Average & 66.14 & 61.68 & 74.45 & 65.53 & 77.49 & 80.19 & 75.71 & 80.36 & $\mathbf{8 1 . 8 1}$ \\
\hline
\end{tabular}

Table 4: Classification accuracies (\%) of training and test data from different sessions

315 achieved similar results with TCA. As compared with these methods, SAAE 


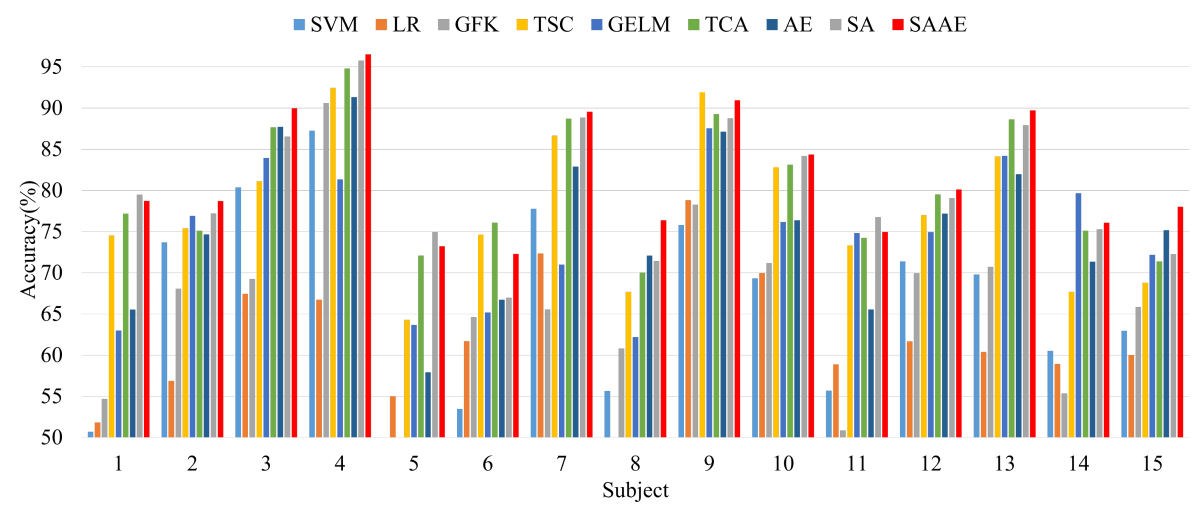

Figure 8: Average classification accuracy (\%) of the six task groups for each participant.

achieved a better performance on all cross-session task groups, because of the non-linearity of the auto-encoder coupled with the subspace alignment solution in the domain-invariant subspace. The average classification accuracy of SAAE on the six task groups for all 15 subjects was $81.81 \%$, an improvement of up to $1.62 \%$ on average as compared to the best baseline TCA. The results show that the improvement for the session-to-session task groups was smaller than that for the subject-to-subject task groups. This phenomenon occurs because the distribution discrepancy between different sessions is much smaller than the subject-to-subject discrepancy. This result verifies that our SAAE is more suitable for tasks with large domain differences. Fig 8 shows that SAAE performed better than TSC in the case of 13 of the 15 subjects, and better than TCA in the case of 14 subjects, which confirms that SAAE can perform stably.

\section{Discussion}

\subsection{Comparison with state-of-the-art methods}

In the previous section, we introduced a novel domain adaptation strategy that combines both nonlinear transformations and consistency constraints in a unified framework. The proposed method achieved a better performance

than state-of-the-art methods on both subject-to-subject and session-to-session 
EEG-based emotion recognition task groups. In this section, we discuss the algorithmic differences of these state-of-the-art methods.

TSC [22] is an algorithm that was inspired by both sparse coding and domain adaptation technology. Specifically, unlike conventional sparse coding, it EEG-based emotion recognition, it still has undesirable features. For any new samples, the proposed domain adaptation strategy must re-train the classifier, because the adaptation matrix mentioned in Section 3.2 must be recalculated. This significantly increases additional computation, and renders our SAAE un385

creates new representations by incorporating nonparametric distance measures into the objective function of sparse coding to constrain the difference of the distribution. As shown in Table 2 Table 3 and Table 4 , TSC remains robust for non-stationary EEG-based emotion recognition. Nevertheless, as a linear transformation, TSC still cannot fully remove the discrepancy completely. TCA 30] is one of the most popular domain adaptation methods used in machine learning for solving computer vision problems. Similar to our SAAE method, TCA and SA without auto-encoder transform features into a subspace with MMD constraints. However, when the domain difference is substantially large, a single transfer matrix is not sufficient to yield a domain-invariant feature space (unlike our deep structure). With the development of deep learning, the domain adaptation strategy based on a stacked auto-encoder [23] has been successfully used to extract common representations from both source and target domains. Deep features can contain abstract concepts that are invariant to domain-specific distributions. However, without consistency constraints, the domains represented by the new deep features may be more distinguishable. The experimental results shown in Table 2, Table 3, and Table 4 demonstrate this drawback.

As compared with the other methods, our SAAE method utilizes the nonlinearity of the auto-encoder coupled with MMD constraints to ensure that the shifted source domain follows a distribution similar to that of the target domain. Although the proposed SAAE method appears to be effective for non-stationary suitable for real-time classification. 


\subsection{Effectiveness analysis}

To compare our SAAE algorithm with other methods more rigorously, we further analyzed its effectiveness by calculating the distribution distance and instance weight of the source domain.

\section{(a)Distribution discrepancy}

Fig 9 (a) shows the distribution of raw EEG features between the training dataset (Session 1) and the testing dataset (Session 2) for Subject 1. The interval between the two sessions was one week or longer. To facilitate visualization, we utilize the first two dimensions to plot the feature distribution. A different feature distribution is found to occur; it is necessary to learn a new domaininvariant feature space to shift the classification boundary. Fig 9 (b) shows that the features from different sessions have been transformed into a domaininvariant subspace by our SAAE method. Clearly, the domain discrepancy of the two sessions has been significantly reduced, such that the training data and test data can easily share a similar classification boundary.

To prove the effectiveness of our SAAE algorithm more rigorously, the MMD distance results computed for several relevant methods on the subject-to-subject $1 \rightarrow 2$ evaluation are given in Table 5. Note that when the MMD distance is smaller, the effectiveness of the domain adaptation algorithm is higher. Without a domain adaptation strategy, the distribution distance of the raw features between training and test datasets is the largest. TCA, the excellent performance of which was shown in Section 4, also has a smaller MMD distance, which indicates that it can explicitly reduce the domain discrepancy. Although the MMD distance generated by AE is worse than that generated by TCA, it is still much smaller than the distribution distance of the raw features. The higher-level representations extracted by the stacked auto-encoder can minimize the domain discrepancy. As expected, our SAAE model shows the minimum distribution distance. Benefiting from the natural nonlinearity and the MMD constraint, SAAE can extract the best representation, i.e., that in which the distribution distance is minimized.

\section{(b)Instance weight}




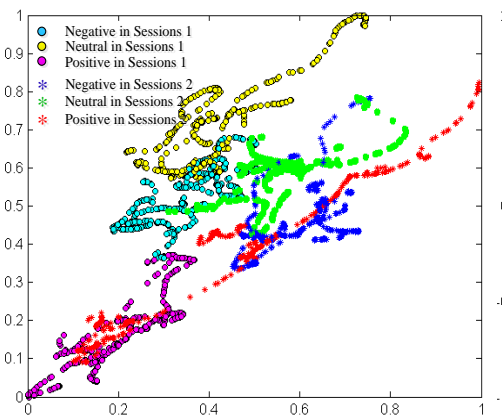

(a) Raw feature

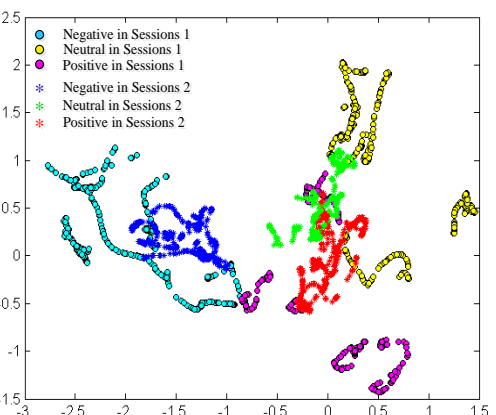

(b) Feature transformed by our SAAE

Figure 9: Feature distributions of the first two dimensions between training and testing sessions for Subject 1.

\begin{tabular}{ccccc}
\hline & SVM & TCA & AE & SAAE \\
\hline Session 1 & 6621 & 152.28 & 3150 & 113.23 \\
Session 2 & 10154 & 80.02 & 2801 & 16.97 \\
Session 3 & 4692 & 97.49 & 3420 & 27.20 \\
\hline
\end{tabular}

Table 5: Maximum mean discrepancy distance results for the subject-to-subject $1 \rightarrow 2$ evaluation

Instance weight is another important indicator of the effectiveness of the proposed method. As mentioned previously, there always exist several source instances that are not relevant to the target instances; increasing the weights of the source instances can help to improve the classification performance. In this study, the nonlinearity of the deep structure made it feasible to shift the training samples with greater importance. To compute the weighting of each instance, we follow the $l_{2,1}$-norm strategy proposed in [35]. In the adaptation matrix mentioned in the subspace alignment section, the $l_{2}$-norm of each row can approximate the importance weighting of each instance in reconstructing the feature representation. To prove the effectiveness of our deep learning strategy, we applied the SAAE method and subspace alignment without an autoencoder to the subject-to-subject $1 \rightarrow 2$ dataset. Fig 10 shows the instance weights results of the two algorithms. Our SAAE method shifts the training samples with greater importance by learning a domain-invariant representation. The significant performance improvement verifies the effectiveness of our deep- 


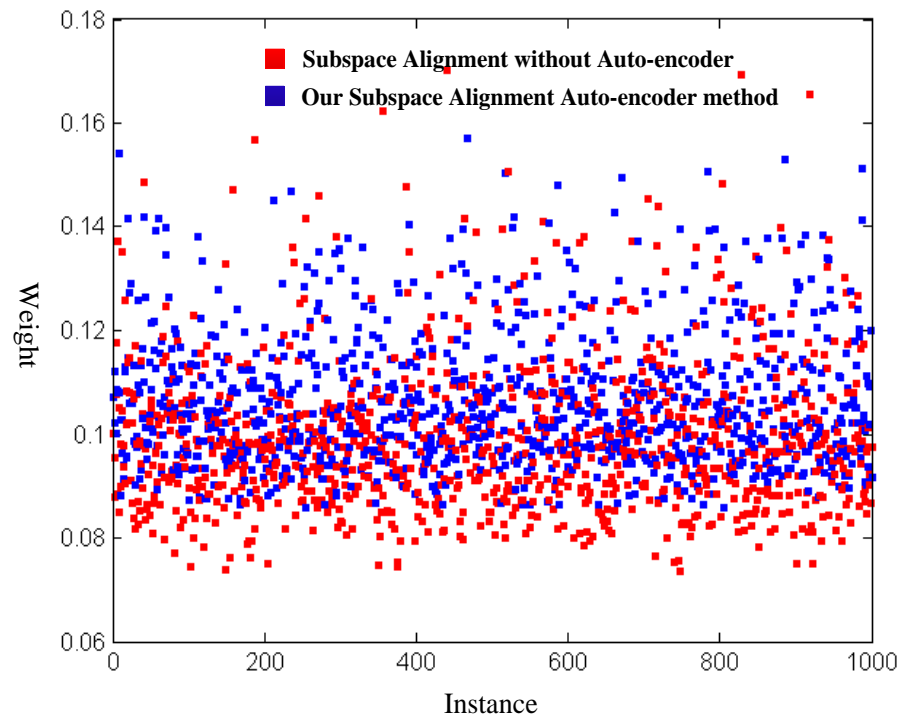

Figure 10: Comparison of instance weight results between our subspace alignment autoencoder method and subspace alignment without an auto-encoder for the subject-to-subject $1 \rightarrow 2$ evaluation

learning-based model.

\subsection{Parameter sensitivity}

There are three key parameters in the proposed SAAE method: the number of hidden neurons $m$, the dimensionality of the second subspace $k$, and the adaptation regularization parameter $\lambda$. The number of hidden neurons is an important parameter for the deep learning method based on the auto-encoder. For comparison, the average classification accuracy of all 14 task groups on the subject-to-subject datasets was computed. When testing a specific parameter, other parameters were fixed. The influence of the parameter $m$ in our SAAE approach is shown in Table 6. The number of hidden neurons should be neither too small (such that the deep representation degenerates), nor too large (such that it increases the computational complexity). In this study, for all experiments, the number of hidden neurons of SAAE was set at 200.

The range of dimensionality of the second subspace $k$ for which the per- 


\begin{tabular}{ccccccc}
\hline Number of hidden neurons & 50 & 100 & 200 & 400 & 600 & 1000 \\
\hline Classification accuracy & 55.79 & 60.43 & 62.50 & 62.33 & 62.42 & 62.25 \\
\hline
\end{tabular}

Table 6: Classification accuracy (\%) of subspace alignment auto-encoder using different numbers of hidden neurons

\begin{tabular}{cccccccc}
\hline Dimensionality of subspace & 20 & 40 & 60 & 80 & 100 & 120 & 150 \\
\hline Classification accuracy & 59.32 & 61.28 & 62.50 & 62.14 & 62.33 & 62.17 & 62.21 \\
\hline
\end{tabular}

Table 7: Classification accuracy (\%) of subspace alignment auto-encoder using different values of dimensionality of the second subspace

formance of SAAE is quite stable is wide, as shown in Table 7 Fixing the dimensionality of the second subspace at 60 results in the best performance for cross-subject emotion classification.

As seen in Table 8, the adaptation regularization parameter $\lambda$ was also investigated. When $\lambda$ is too small, the SAAE optimization problem degenerates, and when $\lambda$ is too large, the domain adaptation method does not perform well. In this study, for all experiments, the adaptation regularization parameter $\lambda$ was set as 1 .

\subsection{Computation time analysis}

In this section, we evaluate the time complexity of most of the classification algorithms compared in Section 4. Because the processes of all the methods mentioned in this paper are similar in the testing stage, we evaluated only the time complexity in the training stage on the dataset of Subject 1 and Subject 2 with 310 features and 6900 samples. Note that the time taken for data preprocessing and feature extraction was not considered. All the algorithms were tested in the same environment using MATLAB R2013b with an Intel core i7$47703.40 \mathrm{GHz}$ processor and $16 \mathrm{~GB}$ of RAM. The results are shown in Table 9.

The computation time of the SVM and LR is much shorter than that of the

\begin{tabular}{cccccc}
\hline$\lambda$ & 0.01 & 0.1 & 1 & 10 & 100 \\
\hline Classification accuracy (\%) & 61.19 & 62.31 & 62.50 & 60.87 & 50.36 \\
\hline
\end{tabular}

Table 8: Classification accuracy (\%) of subspace alignment auto-encoder using different values of the adaptation regularization parameter $\lambda$ 


\begin{tabular}{cccccccc}
\hline & SVM & LR & GFK & TSC & TCA & AE & SAAE \\
\hline Training time $(s)$ & 0.203 & 0.321 & 3.902 & 146.98 & 42.043 & 88.870 & 121.81 \\
\hline
\end{tabular}

Table 9: Time complexity of all baseline methods

465

tation time experiment. As is well known, all the deep-learning-based methods require more time to learn high-level features because of their deep structure.

\section{Conclusion}

In this paper, SAAE, which attempts to learn common representations from non-stationary EEG data across different subjects and sessions, was proposed. In SAAE, an unsupervised auto-encoder learns by both source and target domain features to transform the raw EEG features into a domain-invariant subspace to ensure the feasibility of shifting between domains. To avoid unexpectedly enlarging the cross-domain distribution discrepancy, we constrain the distribution discrepancy by minimizing the MMD in RKHS. Finally, the results of an experiment on EEG datasets for emotion recognition (named SEED) show that the proposed method exhibits a better performance than that of the state-of-the-art methods and verify the effectiveness of the proposed method for decreasing domain discrepancy and reducing performance degradation across subjects and sessions. In the future, we plan to extend the domain-discrepancyreducing strategy to real-time BCI applications.

\section{Acknowledgments}

This project was funded in part by a scholarship from the China Scholarship Council (CSC). We would like to acknowledge the support of CSC. 
This work was supported by the National Natural Science Foundation of China (no. 61402129) and Postdoctoral Foundation Projects (nos. LBH-Z14090 and 2015M571417). Furthermore, Xin Liu was sponsored by the National Natural Science Foundation of China (grant no. 61401117). In addition, Ou Bai was sponsored by the National Science Foundation (USA) (CNS-1552163).

\section{Reference}

[1] N. H. L. C. Y. Chiang, H. M. Hsu, Improving driver alertness through music selection using a mobile eeg to detect brainwaves, Sensors 13(7) (2013) 8199-8221.

[2] F. Sauvet, C. Bougard, M. Coroenne, In flight automatic detection of vigilance states using a single eeg channel, IEEE Transactions on Biomedical Engineering 61(12) (2014) 2840-2847.

[3] C. Muhl, C. Jeunet, F. Lotte, Eeg-based workload estimation across affective contexts, Frontiers in Neuroscience 8(8) (2014) 114-114.

[4] M. Chung, W. Cheung, R. Scherer, R. P. Rao, A hierarchical architecture or adaptive brain-computer interfacing, Proceedings of the, International Joint Conference on Artificial Intelligence, Barcelona, Catalonia, Spain, July (2011) 1647-1652.

[5] T. Zander, S. Jatzev, Context-aware braincomputer interfaces: exploring the information space of user, technical system and environment, Journal of Neural Engineering 9(9) (2012) 16003-16012.

[6] W. L. Zheng, B. L. Lu, Investigating critical frequency bands and channels for eeg-based emotion recognition with deep neural networks., IEEE Transactions on Autonomous Mental Development 7(3) (2015) 162-175.

[7] H. Morioka, A. Kanemura, Learning a common dictionary for subjecttransfer decoding with resting calibration, NeuroImage 111 (2015) 167-178. 
[8] Y. Li, H. Kambara, Y. Koike, Application of covariate shift adaptation techniques in brain-computer interfaces, IEEE transactions on bio-medical engineering 57(6) (2010) 1318-1324.

[9] A. Buttfield, P. W. Ferrez, J. R. Millan, Towards a robust bci: Error potentials and online learning, IEEE Trans. Neural Syst. Rehabil. Eng 14(2) (2006) 164-168.

[10] V. Singh, K. P. Miyapuram, R. S. Bapi, Detection of cognitive states from fmri data using machine learning techniques, Proceedings of the, International Joint Conference on Artificial Intelligence, Hyderabad, India, January (2007) 587-592.

[11] R. Jenke, A. Peer, M. Buss, Feature extraction and selection for emotion recognition from eeg, IEEE Transactions on Affective Computing 5(3) (2014) 327-339.

[12] A. Abraham, F. Pedregosa, Machine learning for neuroimaging with scikitlearn, Frontiers in Neuroinformatics 8(8) (2014) 14.

[13] J. Luo, Z. Feng, J. Zhang, Dynamic frequency feature selection based approach for classification of motor imageries, Computers in Biology and Medicine 75 (2016) 45-53.

[14] S. J. Pan, Q. Yang, A survey on transfer learning, IEEE Transactions on Knowledge and Data Engineering 22(10) (2010) 1345-1359.

[15] M. Long, J. Wang, J. Sun, Domain invariant transfer kernel learning, IEEE Transactions on Knowledge and Data Engineering 27(6) (2015) 1519-1532.

[16] W. L. Zheng, Y. Q. Zhang, J. Zhu, B. L. Lu, Transfer components between subjects for eeg-based emotion recognition, In International Conference on Affective Computing and Intelligent Interaction (2015) 917-922.

[17] W. L. Zheng, B. L. Lu, Personalizing eeg-based affective models with transfer learning, Proc. of the 25th International Joint Conference on Artificial Intelligence (2016) 2732-3738. 
[18] V. Jayaram, M. Alamgir, Y. Altun, Transfer learning in brain-computer interfaces, IEEE Computational Intelligence Magazine 11(1) (2016) 20-31.

[19] M. Shao, D. Kit, Y. Fu, Generalized transfer subspace learning through low-rank constraint, International Journal of Computer Vision 109(1-2) (2014) 74-93.

[20] Y. Shin, S. Lee, M. Ahn, Noise robustness analysis of sparse representation based classification method for non-stationary eeg signal classification, Biomedical Signal Processing and Control 21 (2015) 8-18.

[21] Y. Shin, S. Lee, M. Ahn, Simple adaptive sparse representation based classification schemes for eeg based brain-computer interface applications, Computers in Biology and Medicine 66(C) (2015) 29-38.

[22] M. Long, G. Ding, J. Wang, Transfer sparse coding for robust image representation, IEEE Conference on Computer Vision and Pattern Recognition 9(4) (2013) 407-414.

[23] X. Glorot, A. Bordes, Y. Bengio, Domain adaptation for large-scale sentiment classification: A deep learning approach, International Conference on Machine Learning (2011) 513-520.

[24] J. Deng, Z. Zhang, F. Eyben, Autoencoder-based unsupervised domain adaptation for speech emotion recognition, IEEE Signal Processing Letters 21(9) (2014) 1068-1072.

[25] M. Kan, S. Shan, X. Chen, Bi-shifting auto-encoder for unsupervised domain adaptation, IEEE International Conference on Computer Vision (2015) 3846-3854.

[26] A. Gretton, K. M. Borgwardt, M. Rasch, A kernel method for the twosample-problem, In Advances in Neural Information Processing Systems (2006) 513-520. 
[34] W. L. Zheng, J. Y. Zhu, B. Lu, Identifying stable patterns over time for emotion recognition from eeg, arXiv preprint arXiv 1601.02197.

[35] M. Long, J. Wang, G. Ding, Transfer joint matching for unsupervised domain adaptation, IEEE Conference on Computer Vision and Pattern Recognition (2014) 1410-1417. 